\title{
Le futur diplôme d'études spécialisées (DES) de réanimation en questions-réponses
}

\author{
The future board in intensive care medicine: questions and answers
}

\section{La Commission Jeunes de la Société de Réanimation de Langue Française*}

C SRLF et Springer-Verlag France 2013

\section{Introduction}

La transformation annoncée du diplôme d'études spécialisées complémentaire (DESC) de réanimation médicale en diplôme d'études spécialisées (DES) de réanimation suscite de nombreuses interrogations concernant l'avenir de la spécialité chez les jeunes réanimateurs et chez les étudiants en médecine qui projettent de s'orienter vers la réanimation. Bien qu'aucun calendrier définitif ne soit clairement défini, la Commission Nationale de l'Internat et du Post-Internat (CNIPI), instituée par le décret du 9 mars 2009 afin de proposer une réforme du troisième cycle des études médicales, a récemment remis un rapport (« rapport CNIPI» [1]) qui propose des solutions pour mieux organiser la formation des nombreux internes issus de l'augmentation du numerus clausus. Parmi les mesures phares proposées par ce rapport figure une réforme du DES, avec l'intégration d'une ou deux années de mise en responsabilité. La remise du DES s'effectuerait ainsi chez des médecins totalement formés, puisqu'ayant à leur actif une ou deux années d'exercice en responsabilité, réduisant ainsi le caractère aléatoire du déroulement du post-internat résultant de l'inadéquation entre postes d'internes et postes de chefs de cliniqueassistants dans certaines spécialités. La CNIPI encourage la filiarisation dès le début de l'internat. Ainsi, les anciens DESC de type II deviendraient pour la plupart des spécialités filiarisées. Dans ce cadre, la disparition du DESC de réanimation,

La Commission Jeunes de la Société de Réanimation de Langue Française* $(\bowtie)$

Société de Réanimation de Langue Française,

Maison de la Réanimation, 48, avenue Claude Vellefaux, F-75010 Paris

e-mail : commissionjeunessrlf@gmail.com

\footnotetext{
* Pierre-Marie BERTRAND, Alexandra BEURTON, Alice BLET (secrétaire), Benjamin CLOUZEAU, Laetitia CONTENTIN, Damien CONTOU, Pierre-Éric DANIN, Bruno FILLOUX, Sylvain GARNIER, Élodie GELISSE, Antoine GROS, Vincent PEIGNE (secrétaire adjoint), Nicolas de PROST, Alexis SOUMMER et Florent WALLET
}

comme tous les autres DESC du même type, paraît inéluctable. Il a donc semblé légitime à la Commission Jeunes de la Société de Réanimation de Langue Française (SRLF) de relayer les interrogations des jeunes réanimateurs auprès des doyens de Facultés de médecine impliqués dans cette réforme, afin de faire un état des lieux de ce projet de réforme. Cet article retranscrit, sous forme de questions-réponses, un échange entre les Prs D. Annane (Doyen de l'UFR des sciences de la santé Simone Veil à l'Université de Versailles Saint Quentin et Conseiller pour l'enseignement médical et la recherche au cabinet de la ministre des Affaires Sociales et de la Santé), D. Perrotin (Doyen de la Faculté de médecine de Tours, Président de la Conférence des doyens), B. Schlemmer (Doyen de la Faculté de médecine, Paris-Diderot), A. Vieillard-Baron (Secrétaire général du Collège national des enseignants de réanimation (CNER) représentant F. Fourrier, Président du CNER) et la Commission Jeunes de la SRLF qui s'est tenu à la Maison de la Réanimation le 14 décembre 2012.

Que pensent les étudiants en deuxième cycle d'études médicales de cette réforme?

Les étudiants du deuxième cycle des études médicales sont focalisés sur l'examen classant national (ECN) et ne savent pas à quels DES ils pourront s'inscrire en fonction de leur classement (un écart minime de note résultant en une différence majeure de classement). Malgré la préparation intensive de l'ECN, les étudiants sont peu armés et paradoxalement peu préparés pour faire un choix de DES. Les informer est donc assez difficile. Quand des journées d'information sont organisées par les Facultés, un doyen nous rappelle que seuls 10 à $15 \%$ des étudiants sont présents.

\section{Pourquoi transformer le DESC de réanimation en DES de réanimation?}

La mission interministérielle confiée à la CNIPI et à la CPNES (Commission Pédagogique Nationale des études de santé) pourrait proposer la transformation des DESC de 
type II dits qualifiants en DES et la filiarisation des spécialités. Pour l'instant, pour le régulateur (les pouvoirs publics), la réanimation n'est pas, ou incorrectement, prise en compte dans l'évaluation des besoins démographiques. Le but de la filiarisation est d'aider à la régulation de la formation des professionnels de santé en fonction des données de démographie et de l'évolution de l'offre de soins. Pour le régulateur, le taux de fuite, c'est-à-dire le rapport entre le nombre d'internes entrant dans une filière et le nombre de diplômés exerçant la spécialité est un des facteurs clés de l'équilibre démographique des professions médicales. C'est pourquoi les DESC de type II constituent a priori un facteur de confusion en matière de régulation. Pour ce qui concerne la réanimation, le taux de fuite touche de nombreuses spécialités dont l'anesthésie-réanimation, la pneumologie ou encore la cardiologie. La réanimation, étant par ailleurs d'exercice exclusivement hospitalier, constitue un élément important de l'offre de soins hospitalière. La filiarisation de la réanimation est donc une nécessité en matière d'offre de soins.

\section{Quels sont les besoins dans la spécialité ?}

Le constat fait lors de l'enquête, réalisée par la Fédération de la réanimation et qui fera l'objet d'un numéro spécial de la revue Réanimation, comprenant par ailleurs tous les référentiels de la discipline, montre que les besoins de formation de médecins réanimateurs sont d'environ 1000 pour les dix ans à venir, soit 100 étudiants à former par an pendant les dix prochaines années, sur l'ensemble du territoire [2]. Actuellement, le DESC ne permet pas de répondre à ce besoin car la grande majorité des étudiants inscrits en DESC ne vont pas jusqu'à la validation finale ou retournent à leur spécialité d'origine. Les besoins actuels sont d'environ 70 postes de praticiens hospitaliers publiés chaque année au Journal Officiel (http://www.journal-officiel.gouv.fr/) avec seulement deux tiers des postes pourvus. Il est par ailleurs reconnu dans cette enquête que la formation du DESC est de grande qualité. Il est intéressant de noter que $52 \%$ des inscrits au DESC de réanimation sont issus du DES d'anesthésie-réanimation (DES-AR) selon une enquête réalisée par la Commission Jeunes de la SRLF auprès des étudiants de DESC [3]. Par ailleurs, environ un tiers des médecins qui exercent actuellement en réanimation sont des médecins ayant obtenu la qualification ordinale en réanimation, c'est-à-dire qui ne proviennent ni du DES-AR ni du DESC de réanimation. Il est de la responsabilité des pouvoirs publics de s'assurer que tous les postes nécessaires dans le futur seront pourvus.

\section{Existe-t-il un calendrier prévu pour la mise en application du DES de réanimation ?}

Actuellement, il n'y a pas de date officielle. La dernière élection présidentielle et le changement des équipes responsa- bles ont retardé l'application de la réforme du DES. Cependant, un délai de deux ans pour sa mise en place semble se profiler : l'extension des spécialités filiarisées devant a priori débuter par les DESC de type II qui ont déjà un référentiel métier comme la gériatrie, la chirurgie, les urgences et la réanimation [4]. La CNIPI a été reconduite (décret en cours de publication) et a été missionnée en articulation avec la CPNES pour présenter une méthodologie de réforme du $3^{\mathrm{e}}$ cycle pour septembre 2013 .

\section{Qu'en est-il de la « double compétence » rendue possible par le DESC ?}

Dans la majorité des cas, le choix entre le DES d'origine et le DESC se fait au moment du clinicat. Il est rappelé que le double exercice n'existe pas et est interdit par la loi (principe de l'exercice exclusif des DESC de type II). Chaque médecin doit choisir une spécialité d'exercice unique et ne peut exercer deux spécialités en même temps. De même, il est rappelé que la Validation des Acquis d'Expérience (VAE) continuera d'exister et permettra de ne pas rester enfermé dans une spécialité.

La richesse de la réanimation est basée en partie sur la pluridisciplinarité des DES d'origine des étudiants inscrits en DESC. La filiarisation va, de fait, faire perdre ces différentes origines. Cependant la maquette du futur DES tentera de répondre à ce souhait de conserver " un tropisme » plus prononcé dans un domaine (e.g. cardiologie, pneumologie, néphrologie) ce qui permettrait de garder une polyvalence dans les services telle qu'elle est connue actuellement. Rien n'est à ce jour totalement exclu et ce tropisme pour une autre spécialité pourra éventuellement faire l'objet d'une VAE qui permettra un changement de discipline.

\section{Quelle attractivité pour le futur DES ?}

Il s'agit d'une filiarisation : tous les postes d'interne seront pourvus, même si durant les premières années après la création du DES les étudiants qui choisiront la réanimation ne seront peut-être pas les mieux classés à l'ECN. Le problème de la peur d'une spécialité à gardes dans un contexte de féminisation de la médecine et de changements sociétaux profonds n'est pas propre au DES de Réanimation et concerne également les DES de gynécologie-obstétrique, de chirurgie et le DES-AR, et à plus large échelle tous les médecins, y compris libéraux, qui sont tenus de participer à la permanence des soins.

La réanimation est une spécialité à petits effectifs, mal connue de la population générale et même des étudiants en médecine. Pour améliorer l'attractivité de la spécialité, il est sans doute nécessaire de mieux communiquer sur l'amélioration de la balance vie professionnelle/vie privée (e.g. garde à $18 \mathrm{~h}$, repos de sécurité, application des dix demi-journées 
hebdomadaires...). Il semble également important de souligner qu'il existe des postes disponibles dans des services de réanimation partout en France.

\section{Quels seront les postes ouverts au choix de ce DES de réanimation?}

Les agréments de stage sont donnés par une commission « d'agrément » (dite Commission de subdivision) présidée par les doyens, sur proposition des coordonnateurs locaux de chaque région et les ouvertures de postes d'interne sont réalisées chaque semestre par l'Agence régionale de santé (ARS) lors d'une commission "de répartition" (dite Commission d'évaluation des besoins de formation) avec les coordonnateurs locaux de chaque spécialité et les représentants des internes. La discussion n'est pas propre au DES de réanimation. Il existera nécessairement au niveau local une discussion entre les coordonnateurs de chaque DES puisque d'autres spécialités comme la cardiologie, la médecine interne, la pneumologie, mais aussi l'anesthésieréanimation ont dans leur maquette un/des stage(s) obligatoire(s) ou fortement conseillé(s) en réanimation. Il est de la responsabilité du doyen de chaque faculté d'assurer à chaque étudiant la possibilité de réaliser sa maquette. Donc, localement, des discussions devront être entamées pour définir au cas par cas, pour chaque service de réanimation, combien de postes seront réservés au DES-AR, au DES de réanimation, au futur DES de médecine d'urgence et aux autres DES (spécialités médicales).

\section{Quelle sera l'employabilité du DES de réanimation ?}

La séparation entre réanimation médicale et réanimation chirurgicale semble de plus en plus désuète. Le nombre actuellement élevé des services de réanimation et leur coût très élevé, en termes d'effectifs médicaux et paramédicaux, vont entraîner une tendance au regroupement des services pour des services de réanimation médicochirurgicale ou polyvalente dans les structures de moyenne taille et une disparition de ces services dans les structures de plus petite taille. Une restructuration des hôpitaux dans les dix ans à venir va très probablement s'effectuer avec un besoin de médecins poly- valents pour faire fonctionner des services de réanimation et de surveillance continue de grande taille. L'articulation entre anesthésie et réanimation sera peut-être ainsi amenée à évoluer du fait de l'évolution de la démographie médicale, de la réorganisation hospitalière et de la nécessité de préserver l'activité opératoire.

\section{Conclusion}

La transformation du DESC de réanimation en DES de réanimation apparaît inéluctable car elle s'inscrit dans le cadre d'une réforme des DES qui dépasse largement le cadre de la spécialité réanimation. Même si certains aspects spécifiques à la réanimation, comme la question de la traditionnelle « double compétence », restent en suspens, cette réforme devrait apporter plus de visibilité à la spécialité et permettre de remplir les objectifs quantitatifs de formation de réanimateurs, condition sine qua non du développement de notre discipline.

Remerciements La Commission Jeunes de la SRLF remercie Djillali Annane, Dominique Perrotin, Benoît Schlemmer et Antoine Vieillard-Baron pour leur aide.

Conflit d'intérêt : les auteurs déclarent ne pas avoir de conflit d'intérêt.

\section{Références}

1. Rapport de la Commission Nationale de l'Internat et du PostInternat

2. Fédération de la Réanimation, Annane D, Diehl JL, Drault JN, et al (2013) Démographie et structure des services de réanimation français (hors réanimation chirurgicale) : état des lieux. Réanimation [in press]

3. Commission Jeunes de la SRLF (2012) Les questions que se posent les étudiants du DESC de réanimation. Journée nationale du DESC de réanimation médicale : jeudi 9 février 2012 Maison de la Réanimation

4. Comité de coordination de la réanimation (2011) Référentiel de compétences et d'aptitudes du médecin réanimateur. Réanimation 20(Suppl 3):S705-S24 